\title{
Stimulus-Induced and State-Dependent Sustained Gamma Activity Is Tightly Coupled to the Hemodynamic Response in Humans
}

\author{
Stefan P. Koch, ${ }^{1}$ Peter Werner, ${ }^{1}$ Jens Steinbrink, ${ }^{1}$ Pascal Fries, ${ }^{2}$ and Hellmuth Obrig ${ }^{1,3}$ \\ ${ }^{1}$ Berlin NeuroImaging Center, Charité-Universitätsmedizin Berlin, 10117 Berlin, Germany, ${ }^{2}$ Donders Institute for Brain, Cognition, and Behaviour, \\ Radboud University Nijmegen, 6500 HB Nijmegen, The Netherlands, and ${ }^{3}$ Max Planck Institute for Human Cognitive and Brain Sciences, Leipzig, \\ and Day Clinic of Cognitive Neurology, University of Leipzig, 04103 Leipzig, Germany
}

A prompt behavioral response to a stimulus depends both on the salience of the stimulus as well as the subject's preparedness. Thus, both stimulus properties and cognitive factors, such as attention, may determine the strength of neuronal synchronization in the gamma range. For a comprehensive investigation of stimulus-response processing through noninvasive imaging, it is, however, a crucial issue whether both kinds of gamma modulation elicit a hemodynamic response. Here, we show that, in the human visual cortex, stimulus strength and internal state modulate sustained gamma activity and hemodynamic response in close correspondence. When participants reported velocity changes of gratings varying in contrast, gamma activity $(35-70 \mathrm{~Hz})$ increased systematically with contrast. For stimuli of constant contrast, the amplitude of gamma activity before the behaviorally relevant velocity change was inversely correlated to the behavioral response latency. This indicates that gamma activity also reflects an overall attentive state. For both sources of variance, gamma activity was tightly coupled to the hemodynamic response measured through optical topography. Because of the close relationship between high-frequency neuronal activity and the hemodynamic signal, we conclude that both stimulus-induced and statedependent gamma activity trigger a metabolic demand and are amenable to vascular-based imaging.

\section{Introduction}

Local field potentials (LFPs) in the gamma range are an auspicious electrophysiological candidate measure of stimulus-related and top-down processing (Eckhorn et al., 1988; Gray and Singer, 1989; Gray et al., 1989). Noninvasive neuroimaging, however, primarily relies on the vascular response. Thus, the question arises of how neuronal synchronization evokes a vascular response. Recently, a close coupling between gamma oscillations and the hemodynamic response was reported in the cat's visual cortex (Niessing et al., 2005). Increases in visual contrast yielded stronger gamma synchronization and a larger vascular response. Thus, in the animal, gamma band and hemodynamics likewise indicate externally driven brain responses. Interestingly, also for a given contrast, both signals fluctuated while the tight coupling between neuronal and vascular response was preserved. This indicates that both responses are modulated by state-dependent variables. Highlighting this state dependence, trained monkeys show a clear augmentation of gamma-band LFP when attending

Received March 24, 2009; revised Aug. 20, 2009; accepted Aug. 24, 2009.

This work was supported by grants from the Bundesministerium für Bildung und Forschung (Berlin Neurolmaging Center and Bernstein Center for Computational Neuroscience Berlin and Grant FK-01GZ0710) and European Union Grants nEUROpt-201076 and NEST-012778.

Correspondence should be addressed to Stefan P. Koch, Berlin Neurolmaging Center/Department of Neurology Charité Campus Mitte, Universitätsmedizin Berlin, Charitéplatz 1, 10117 Berlin, Germany. E-mail: stefan.koch@ charite.de.

DOI:10.1523/JNEUROSCI.1402-09.2009

Copyright $\odot 2009$ Society for Neuroscience $\quad$ 0270-6474/09/2913962-09\$15.00/0 to a stimulus compared with the unattended perception of the same stimulus (Womelsdorf et al., 2006).

Although variation in internal states such as attention cannot be easily monitored in the anesthetized animal, reaction time (RT), a measure concatenating both externally and internally generated response determinants, allows for a differentiation between stimulus-related and state-dependent variables in humans. Here, we combine noninvasive EEG and optical topography to simultaneously assess gamma-band and cortical oxygenation changes. Optical topography is a noninvasive extension of intrinsic optical signals as used in the study in the cat's visual cortex (Niessing et al., 2005). Since focal decreases in the concentration of deoxygenated hemoglobin are the source of blood oxygen level-dependent (BOLD) contrast increases, results using optical topography are applicable to the large body of functional magnetic resonance imaging studies in humans (Kleinschmidt et al., 1996; Huppert et al., 2006).

In the present study, subjects had to report unpredictable changes in velocity of a moving grating whose contrast changed parametrically (see Fig. $1 A$ ) but was irrelevant for the task. Thus, salience of the stimulus was altered by external modulation (contrast) independent of the cue for behavioral response (velocity). We hypothesized that increasing salience would decrease reaction time. The variability in reaction time for a fixed contrast additionally allowed investigation of the relationship between the subject's "preparedness" and the magnitude of gamma activity. In our combined EEG/optical-topography approach, we therefore assessed (1) stimulus-dependent modulations and (2) endogenous 

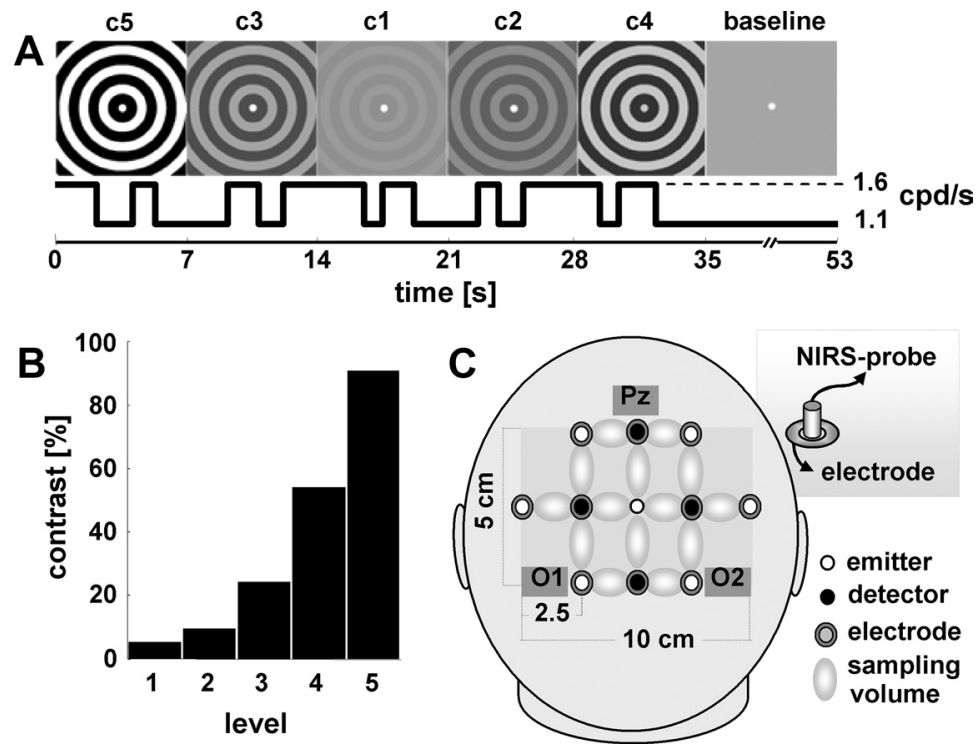

Figure 1. Experimental design. $\boldsymbol{A}$, Concentrically moving gratings changed contrast $(c 1-c 5)$ every $7 \mathrm{~s}$ in a pseudorandomized order. Within each $7 \mathrm{~s}$ period, the subject had to detect and respond to three changes in velocity, which alternated between 1.1 and $1.6 \mathrm{cpd} / \mathrm{s}$. Because contrast and velocity were modulated independently, external modulation of stimulus salience (contrast) and additional internal response determinants could be disentangled by means of reaction time. An $18 \mathrm{~s}$ baseline period, for which contrast was kept at $0 \%$, was inserted every $35 \mathrm{~s}$ (i.e., after 5 contrast changes). $\boldsymbol{B}$, Michelson contrasts of the gratings tested (c1-c5). C, Sketch of the probe arrangement over the occipital-parietal region. Optical probes were inserted into electrode rings (inset), allowing for simultaneous measurements of electrophysiological and oxygenation response over the same area.

fluctuations of stimulus processing as reflected in the gammaband response (GBR). Additionally, we investigated (3) the relationship between both types of GBR and the simultaneously assessed hemodynamic response.

The need to experimentally demonstrate that both statedependent and stimulus-driven GBR changes are reflected in the vascular response results from increasing evidence for substantial deviations from a straightforward neurovascular coupling (Caesar et al., 2003; Sirotin and Das, 2009). This also applies to macroscopically derived coupling between slow oscillatory activity and the vascular response (Goldman et al., 2002; Moosmann et al., 2003; Koch et al., 2006). Hence it cannot be easily assumed that stimulusdriven GBR changes will create the same metabolic demand as changes in gamma synchronization related to top-down processes (Fries et al., 1997).

On a conceptual level, it has been proposed that attention enhances perceptual sensitivity. In animals, V4 and MT neurons respond similarly to an increase in "physical" salience and to an increase in attention (Martínez-Trujillo and Treue, 2002; Reynolds and Desimone, 2003; Reynolds and Chelazzi, 2004). There is also evidence that attention increases the contrast perception (Carrasco et al., 2004). The increase of contrast sensitivity and spatial resolution by attention would indeed speak for a mechanism that is latched at a given processing level and mimics an increase in stimulus salience. Whether the underlying networks, eliciting large-scale GBR attributable to stimulus-driven as opposed to state-dependent modulation are identical cannot be differentiated by noninvasive techniques applied here and is beyond the scope of this paper.

\section{Materials and Methods \\ Participants}

Twelve participants (mean age, 24.3 years; range, 20-35 years; six females) were included in the study. Participants had normal or correctedto-normal visual acuity, were neurologically and otherwise healthy, gave written informed consent, and were financially compensated for partic- ipation. The study was approved by the local ethics committee (Charité; Universitätsmedizin Berlin).

Experimental design and visual stimuli A concentrically moving grayscale circular whole-field grating (spatial frequency, $1 \mathrm{cpd}$ ) was presented during the complete acquisition period. Every $7 \mathrm{~s}$, the contrast of the grating varied pseudorandomly between five different isoluminant contrasts (Michelson contrast, 5, 9, 24, 54, 91\%) (Fig. 1B) (mean luminance, 98 $\mathrm{cd} / \mathrm{m}^{2}$ ). A baseline condition of $18 \mathrm{~s}$ was inserted after every fifth trial ( $0 \%$ Michelson contrast, i.e., isoluminant static gray screen). In total, each contrast level was presented 70 times, whereas baseline trials appeared 50 times. To minimize fatigue and adaptation effects, the experiment was run in eight blocks. Within each contrast trial, three small velocity changes of the grating occurred at an unpredictable moment. Velocity was toggled between 1.1 and $1.6 \mathrm{cpd} / \mathrm{s}$ and was counterbalanced across all contrasts. To avoid transfer effects from previous contrast trials, the first velocity change appeared randomly between 1.5 and $2.5 \mathrm{~s}$ after trial onset, whereas the third velocity change occurred not less than $1.2 \mathrm{~s}$ before the next contrast change. Participants were asked to fixate a small central fixation point during the full length of the trials and respond by a button press. Acceleration required the right index finger response, whereas deceleration required the left; after four blocks, the hand velocity assignment was reversed. To improve behavioral performance, each block ended with a visual feedback regarding mean reaction time and correctness of response for the last two blocks. Stimuli were presented on a 19 inch cathode ray tube monitor ( $100 \mathrm{~Hz}$ refresh rate) placed $75 \mathrm{~cm}$ in front of the subject. The experimental paradigm was performed with Matlab (Mathworks), based on the Cogent Toolbox (Wellcome Department of Imaging Neuroscience, London, UK). The recording session lasted $\sim 75 \mathrm{~min}$.

\section{Data acquisition}

EEG

EEG was recorded from 32 channels placed according to the 10-20 system (BrainAmp; Brain Products; Easycap; Falk Minow Services). Electrode impedances were kept at $<5 \mathrm{k} \Omega$; FCz served as a reference. Data were filtered between 0.01 and $250 \mathrm{~Hz}$ and sampled at $1000 \mathrm{~Hz}$. Horizontal and vertical electro-oculograms (EOGs) were registered with four additional electrodes.

\section{Optical topography}

Changes in cortical hemoglobin oxygenation were measured concurrently with EEG over the parieto-occipital region with an optical topography system (ISS, Imagent; $10 \mathrm{~Hz}$ sampling frequency). Because light in the near-infrared range penetrates biological tissue rather well, optical spectroscopy of the cerebral cortex is feasible when used on the adult human head (Obrig and Villringer, 2003). The methodology renders concentration changes in oxygenated $(\mathrm{HbO})$ and deoxygenated hemoglobin (HbR), which can be quantified under the assumption of specific and constant optical background properties. The optical topograph used here emits light at 760 and $830 \mathrm{~nm}$, which is guided to the head by fiber optic bundles (diameter, $0.4 \mathrm{~mm}$ ). The reflected light is collected by another set of optic probes (diameter, $3 \mathrm{~mm}$ ) at a distance of $2.5 \mathrm{~cm}$ and amplified by photon multipliers. The system uses four sources and eight detectors arranged in a rectangular array, thus supplying a rough topographical image from 14 measuring volumes over the parieto-occipital cortex. The optical probes were mounted into electrode rings, covering the coregistered EEG electrodes P1/2, Pz, Po3/4 PO7/8, O1/2, and $\mathrm{Oz}$ (Fig. 1C). 


\section{Data analysis}

Effect of the parametrically varied Michelson contrast

EEG. Off-line analysis of the EEG and near-infrared spectroscopy (NIRS) data was performed with Matlab. EEG data were bandpass filtered (0.3$120 \mathrm{~Hz}$ ) and downsampled to $250 \mathrm{~Hz}$. Data were segmented for additional analysis. For each contrast trial, the segment started $1 \mathrm{~s}$ before and ended $8 \mathrm{~s}$ after each contrast change. Baseline trials were segmented into four epochs of $4 \mathrm{~s}$ starting $1 \mathrm{~s}$ after the presentation of the gray screen. Data epochs contaminated by eye movements, muscle activity, or line artifacts were discarded using semiautomatic artifact rejection routines. All epochs were visually reinspected for artifacts. Time-frequency representations (TFRs) were calculated using wavelet analysis (Morlet; nine cycles; frequency range, $4-100 \mathrm{~Hz}$; resolution, $1 \mathrm{~Hz}$ ) to estimate the spectral activity for baseline and each contrast condition. The procedure provides a continuous measure of the amplitude of frequency components (Tallon-Baudry and Bertrand, 1999). Wavelet analysis was calculated for each trial individually. For baseline and each contrast condition, the resulting TFRs were averaged across trials. TFRs were expressed as changes relative to the baseline (Henrie and Shapley, 2005; Hoogenboom et al., 2006) (i.e., for each contrast condition, electrode, and subject, the activity of each frequency bin of the respective TFR was divided by the mean baseline activity of this frequency). To obtain a measure for each frequency band, relative changes were averaged in time (from 0.5 to $6.5 \mathrm{~s}$ after onset) and frequency (theta, $4-7 \mathrm{~Hz}$; alpha, 8-13 Hz; beta, 15-25 $\mathrm{Hz}$; gamma, 35-70 Hz). These values were pooled for the occipital electrodes (O1 and $\mathrm{O} 2)$.

Optical topography. Concentration changes of $\mathrm{HbO}$ and $\mathrm{HbR}$ were calculated using the modified Beer-Lambert law. To attenuate pulserelated changes, data were low-pass filtered (Butterworth; third order; cutoff, $0.3 \mathrm{~Hz}$ ). Channels were visually inspected for artifacts. Brief movement artifacts were corrected by a linear interpolation of the first and the last data points surrounding the artifacts. To obtain the hemodynamic response to each Michelson contrast, a design matrix consisting of five $0-1$ boxcar predictors was convoluted with a hemodynamic response function with a peak at $5 \mathrm{~s}$ (Boynton et al., 1996). This model and the data were fed into a general linear model (GLM). To improve the model, the predictor matrix of the GLM comprised an additional cosinefilter matrix to deal with drifts and slow oscillatory changes (low cutoff at $0.033 \mathrm{~Hz}$ ). Because the experimental design included a baseline trial only after every fifth trial, and because of the sluggishness of the hemodynamic response, the responses to individual trials partly overlapped. As long as the interstimulus interval does not fall below several seconds, the hemodynamic responses to temporally adjacent events can be described as a linear time-invariant system (Boynton et al., 1996). To extract the time courses of the response to each contrast (see Fig. 4), a pseudodeconvolution technique was applied (Koch et al., 2006): for each contrast level, the GLM-derived $\beta$ value of the respective condition was set to zero while all other $\beta$ values remained unchanged. Afterward, the $\beta$ values were multiplied with the predictor matrix and subtracted from the real data. This analytical step results in a time course with the length of the experimental session that approximates the effect of the respective contrast alone, minimizing the hemodynamic effects of all other contrasts. To obtain time courses for $\mathrm{HbO}$ and $\mathrm{HbR}$, the hemodynamic response from 0 to $20 \mathrm{~s}$ after stimulus onset was averaged for each contrast and channel (see Fig. 4C,D). Baseline correction was applied before averaging using the first $0.5 \mathrm{~s}$ after stimulus onset as a reference. NIRS data of the two occipital channels corresponding to the EEG electrodes O1 and O2 were pooled. Repeated-measure ANOVAs were performed for EEG bands and hemodynamic responses separately. Post hoc paired $t$ tests (Bonferroni corrected) were applied if an ANOVA revealed a significant effect.

\section{Internal state-dependent effect}

$E E G$. Single-trial analysis was performed for the highest contrast (c5, 91\% Michelson contrast). EEG data of c5 were segmented from $0.8 \mathrm{~s}$ before to $0.8 \mathrm{~s}$ after the onset of each velocity change. Of the resulting 210 trials, only trials with a behavioral response within $250-700 \mathrm{~ms}$ were considered as valid trials. TFRs were calculated using the same approach as for the contrast effect and expressed as relative change compared with baseline. For each subject, TFRs were sorted according to the response time and grouped with equal size (one-third of the trials) into fast, medium, and slow response classes. For each response group, TFRs were averaged in the time window $200-100 \mathrm{~ms}$ before the onset of the velocity change and across the frequency dimension corresponding to gamma band activity $(35-70 \mathrm{~Hz})$. These values were pooled for the occipital electrodes (O1 and $\mathrm{O} 2)$.

Optical topography. The analysis of the hemodynamic data followed the steps described above except that single-trial time courses for the highest contrast were not averaged. The extraction of single-trial hemodynamic responses for events following in rapid succession is critical especially if the exact individual hemodynamic response function is not known. In this case, the hemodynamic response of an event can be seriously confounded by the influence of adjacent events. Therefore, another approach was chosen: the same hemodynamic response was ascribed to the three respective behavioral responses within one trial of contrast c5. Next, the time courses were sorted according to the behavioral responses. The rationale of the approach is that the hemodynamic responses should be distributed uniformly across the RT dimension if no underlying relationship exists between reaction time and hemodynamic response. Time courses that did not match the criteria for a correct response (see above) were skipped. Again, NIRS time courses were grouped into fast, medium, and slow RT classes (one-third of the total trial number, equally large in size) and averaged. EEG data were averaged across $\mathrm{O} 1$ and $\mathrm{O} 2$. In correspondence to EEG, the two channels overlying $\mathrm{O} 1$ and $\mathrm{O} 2$ were averaged. Differences between fast and slow responses were tested with paired $t$ tests.

\section{Results}

\section{Externally driven response variance by stimulus contrast}

Subjects were instructed to concentrate on a concentrically moving grating of different contrast levels and press a button after a small velocity change of the grating. As expected, across subjects, mean reaction time and error rate decreased with increasing contrast (Fig. 2A,B). With respect to the EEG frequency bands, we found that occipital gamma power (pooled electrodes $\mathrm{O} 1$ and O2) parametrically increased with stimulus contrast (Fig. $2 F$ ). None of the other frequency bands (theta, alpha, beta range) varied systematically with contrast (Fig. 2C-E; supplemental Fig. 1 , available at www.jneurosci.org as supplemental material). One-way repeated measures ANOVAs confirmed the finding of a significant contrast effect exclusively for the gamma band (gamma: $F_{(4,44)}=12.962, p<0.001$; theta: $F_{(4,44)}=1.869, p=0.133$; alpha: $F_{(4,44)}=1.382, p=0.256$; beta: $\left.F_{(4,44)}=1.407, p=0.248\right)$. Post hoc $t$ tests revealed significant differences between contrast conditions for gamma power, and tests of within-subjects contrasts indicate a positive linear relationship $\left(F_{(1,11)}=18.773 ; p=\right.$ 0.001 ) between contrast level and gamma power (supplemental Table 1, available at www.jneurosci.org as supplemental material). No systematic increase of gamma-band activity with contrast strength was found for the channels monitoring ocular movements (EOG) (supplemental Fig. 2, available at www. jneurosci.org as supplemental material). Gamma activity was tonically enhanced over the entire $7 \mathrm{~s}$ stimulation period for each contrast (exemplary single subject data depicted in Fig. $3 A$ ). Thus, the measured activity in the gamma range is not attributable to a phasic response to the onset of the contrast change or fluctuations of attention but reflects an externally driven tonic response to the ongoing stimulus.

\section{Coupling between gamma activity and hemodynamic response}

As suggested by a report on an invasive approach (Niessing et al., 2005), we next addressed the question whether in humans the hemodynamic response would also be linked to the observed tonic changes in externally driven gamma changes. Data from pooled occipital probes (colocated to electrodes $\mathrm{O} 1$ and $\mathrm{O} 2$ ) 

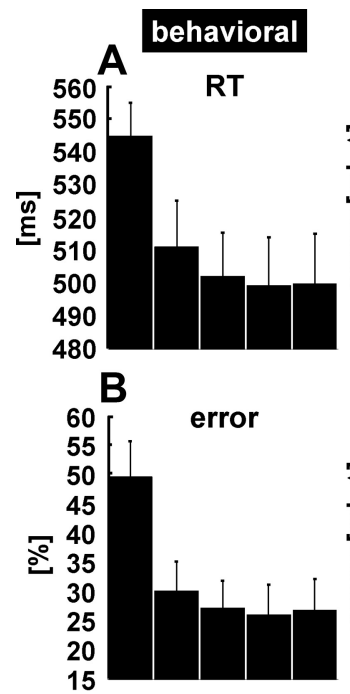
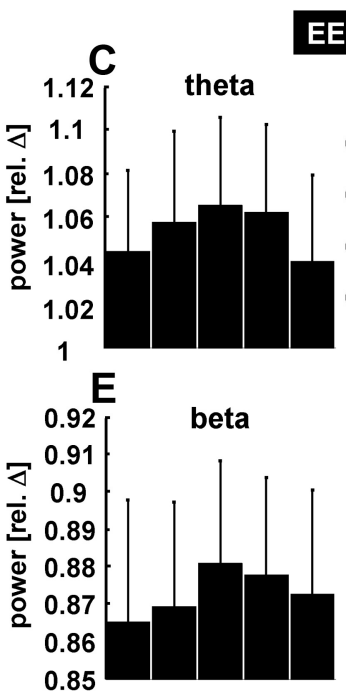

EEG

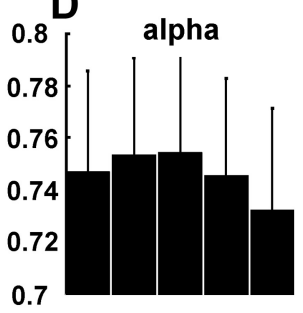

$\mathbf{F}$

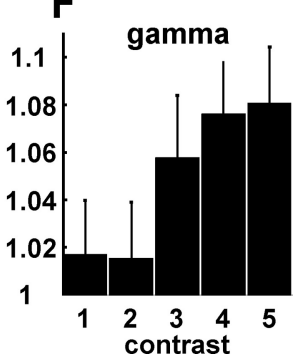

Figure 2. Behavioral and neuronal response to contrast variation across subjects. $A, B$, Mean reaction time (in milliseconds) $(\boldsymbol{A})$ and error rate (in percentage) $(\boldsymbol{B})$ decrease with increasing contrast strength. $\boldsymbol{C}-\boldsymbol{F}$, Changes of oscillatory power in different frequency bands: theta $(4-7 \mathrm{~Hz})(\boldsymbol{C})$, alpha $(8-13 \mathrm{~Hz})(\boldsymbol{D})$, beta $(15-25 \mathrm{~Hz})(\boldsymbol{E})$, and gamma $(35-70 \mathrm{~Hz})(\boldsymbol{F})$ band. 0scillatory power was averaged from 0.5 to $6.5 \mathrm{~s}$ after trial onset from pooled occipital electrodes 01 and 02 . The data are depicted as relative power changes for each frequency band with respect to the baseline (value of 1). Values $<1$ correspond to a decrease in power, whereas values $>1$ represent an increase in power; error bars represent SEM. Compared with the baseline, alpha and beta activity are desynchronized, whereas theta and gamma activity are synchronized during stimulation. Only gamma band activity shows a systematic increase in power with increasing contrast. The vertical bars denote SEM across subjects.

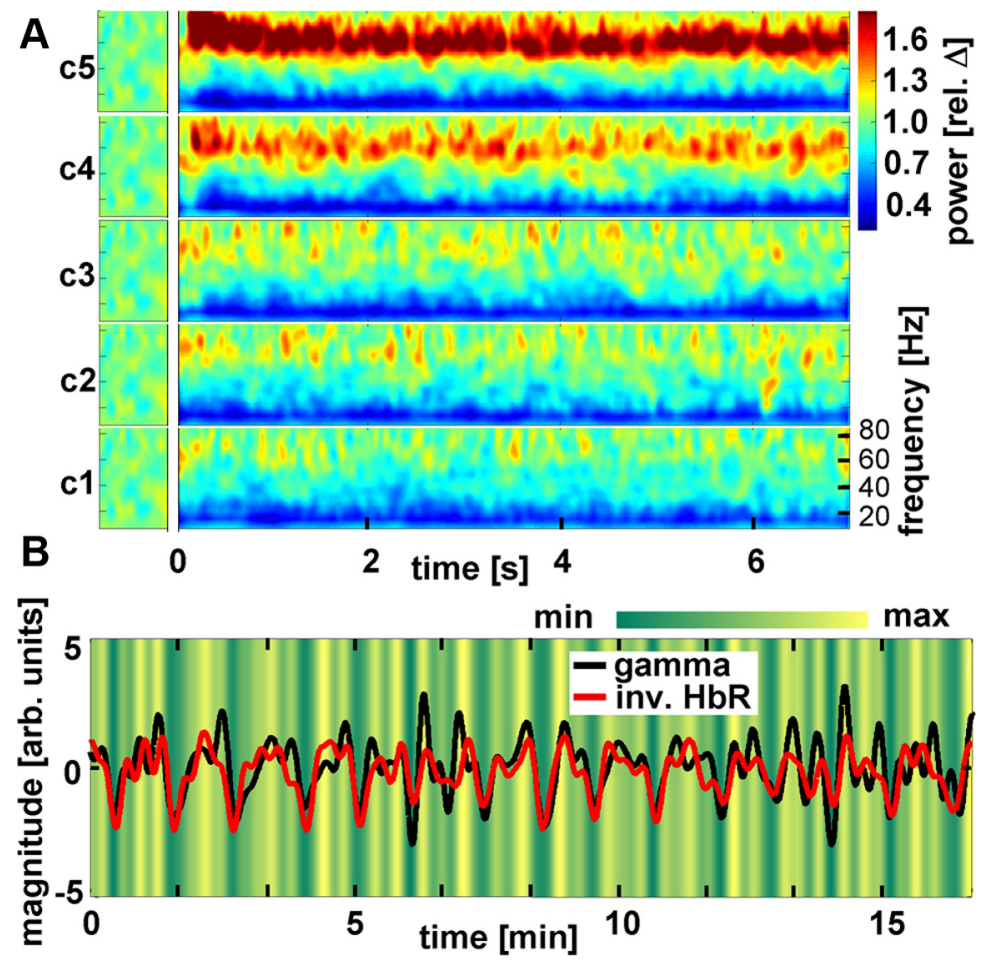

Figure 3. Electrophysiological and oxygenation response to contrast changes. $\boldsymbol{A}$, Time-frequency representations averaged over all $7 \mathrm{~s}$ segments of each contrast from a single subject (pooled electrodes 01 and 02 ) showing a response in the upper gamma frequency range ( $\sim 75 \mathrm{~Hz})$. GBR is strongest for the highest contrast and decreases with lower contrasts. Note that gamma activity is sustained over the full length of stimulation. B, Fluctuations of occipital gamma activity (black; pooled electrodes 01 and 02 ) and $\mathrm{HbR}$ (red; pooled from colocated occipital probes) from a single subject for a period of $\sim 16 \mathrm{~min}$. The background shading (yellow- green) depicts the changes in contrast strength. The close coupling between gamma and $\mathrm{HbR}$ changes is clearly seen in the unaveraged data; both parameters follow the contrast changes showing highest values for the largest contrast (yellow shading) and lowest values for the $0 \%$ contrast baseline (dark green shading). Note that contrast changes and gamma power are convolved with a HRF to allow for a comparison with the hemodynamic response. revealed that oxygenation increased with gamma power when the latter is convolved with a hemodynamic response function (HRF). As an example, Figure $3 B$ depicts the fluctuation of gamma power and oxygenation changes in a single subject over the duration of $\sim 16 \mathrm{~min}$. It can be seen that electrophysiological activity in the gamma range and the hemodynamic response are both driven by stimulus contrast. The grand average across all subjects from occipital probes (colocated to electrodes $\mathrm{O} 1$ and $\mathrm{O} 2$ ) confirmed the finding, yielding a steady increase in the magnitude of the oxygenation response with contrast (Fig. $4 A$ for the increase in $\mathrm{HbO}$; Fig. $4 B$ for $\mathrm{HbR}$; note a decrease in $\mathrm{HbR}$ signals an increase in oxygenation) (supplemental Fig. 1, available at www. jneurosci.org as supplemental material). One-way repeated-measures ANOVAs revealed a significant effect of contrast modulation for $\mathrm{HbO}\left(F_{(4,44)}=6.268 ; p<0.001\right)$ and $\operatorname{HbR}\left(F_{(4,44)}=11.942 ; p<0.001\right)$ (for post hoc $t$ tests of $\mathrm{HbR}$, see supplemental Table 1, available at www.jneurosci.org as supplemental material). Tests of withinsubjects contrasts indicated significant linear trends for $\mathrm{HbO}\left(F_{(4,44)}=6.268 ; p<\right.$ $0.001)$ and $\operatorname{HbR}\left(F_{(1,11)}=8.917 ; p=\right.$ $0.012)$. Furthermore, the averaged time courses of $\mathrm{HbR}$ and $\mathrm{HbO}$ represent a typical hemodynamic response and validate the gradual effect of the contrast strength (Fig. 4C,D).

To test whether electrophysiological and vascular responses were driven by stimulus contrast, we additionally analyzed the correlation between the five Michelson contrasts and the dependent neuronal and hemodynamic signals. As hypothesized, occipital gamma power and HbR correlated well with Michelson contrast (Fig. 5A). The best prediction for this correlation was obtained when applying a logarithmic model (gamma: $F_{(1,3)}=$ 46.6125, $R^{2}=0.940, p=0.006$; with model coefficients $\mathrm{b} 0=-0.72$ and $\mathrm{b} 1=$ 0.397; HbR: $F_{(1,3)}=67.666, R^{2}=0.958$, $p=0.004$; with model coefficients $\mathrm{b} 0=$ -0.462 and $\mathrm{b} 1=0.336$ ). Thus, gamma activity and HbR logarithmically increased with Michelson contrast, whereas the relationship between gamma activity and hemodynamic response is best explained by a linear model $\left(F_{(1,3)}=22.788\right.$, $R^{2}=0.884, p=0.017$; with model coefficients $b 0=0.001$ and $b 1=-0.002$ ).

In sum, as opposed to the lower EEG frequency bands, occipital gamma band activity varies parametrically with stimulus contrast. Furthermore, this systematic variation in gamma power is tightly cou- 
pled to the colocated hemodynamic response. We find a logarithmic increase in both parameters with Michelson contrast, resulting in a linear correlation between neuronal and vascular response. Additionally we analyzed the correlation between the transient response [i.e., the visual evoked potential (VEP) elicited by the onset of each contrast]. The P100 component of the grand average VEP (pooled electrodes $\mathrm{O} 1$ and $\mathrm{O} 2$ ) also increased with stimulus contrast and revealed a strong correlation with both the GBR and the vascular response (supplemental Fig. 3, available at www.jneurosci. org as supplemental material).

\section{Internal state-dependent response variance}

So far, we have shown that gamma activity and vascular responses are coupled when they are driven by external parameters (i.e., the salience of the stimulus). However, invasive experiments in the monkey have shown that gamma power is also strongly modulated by attention (Womelsdorf et al., 2006). Hence, we next analyzed neuronal response variability originating from internal state-dependent parameters. To this end, all behavioral responses to the velocity change were grouped with respect to reaction time (mean RTs: fast, 395.9 ms; medium, $492.4 \mathrm{~ms}$; slow, $611.1 \mathrm{~ms}$ ). This analysis was performed for the highest Michelson contrast (c5; 91\%) to minimize confound by error rate. The approach rests on the fact that behavioral response variability stems not only from differences in stimulus and task properties, but also depends on the instantaneous, internal state of the brain, which has been shown to fluctuate substantially (Arieli et al., 1996). Indeed, when the contrast was kept constant, occipital gamma power (pooled $\mathrm{O} 1$ and $\mathrm{O} 2$ electrodes) before the velocity changes differed substantially for fast, medium, and slow reaction times across subjects (Fig. 6A). Both occipital time-frequency representations across the full frequency range (top panel) and the time course of averaged gamma power $(35-70 \mathrm{~Hz}$; bottom panel) demonstrated that mean reaction time is inversely related to the grand average gamma power before the velocity change. When collapsed over a time window from 200 to $100 \mathrm{~ms}$ before the onset of the velocity change (Fig. $6 \mathrm{~B}$ ), fast reaction times were preceded by stronger gamma activity compared with medium and slow RTs. Paired $t$ tests confirmed significant differences in gamma-band activity between the fast and slow RT trials $\left(t_{(11)}=2.871 ; p=0.015\right)$. In comparison, for the same temporal window using the same band windows as defined for the analysis of contrast strength, the lower frequency bands revealed no significant power differences between fast and slow RT trials at the occipital region of interest $(\mathrm{ROI})$ (theta: $t_{(11)}=0.409, p=0.691$; alpha: $t_{(11)}=$
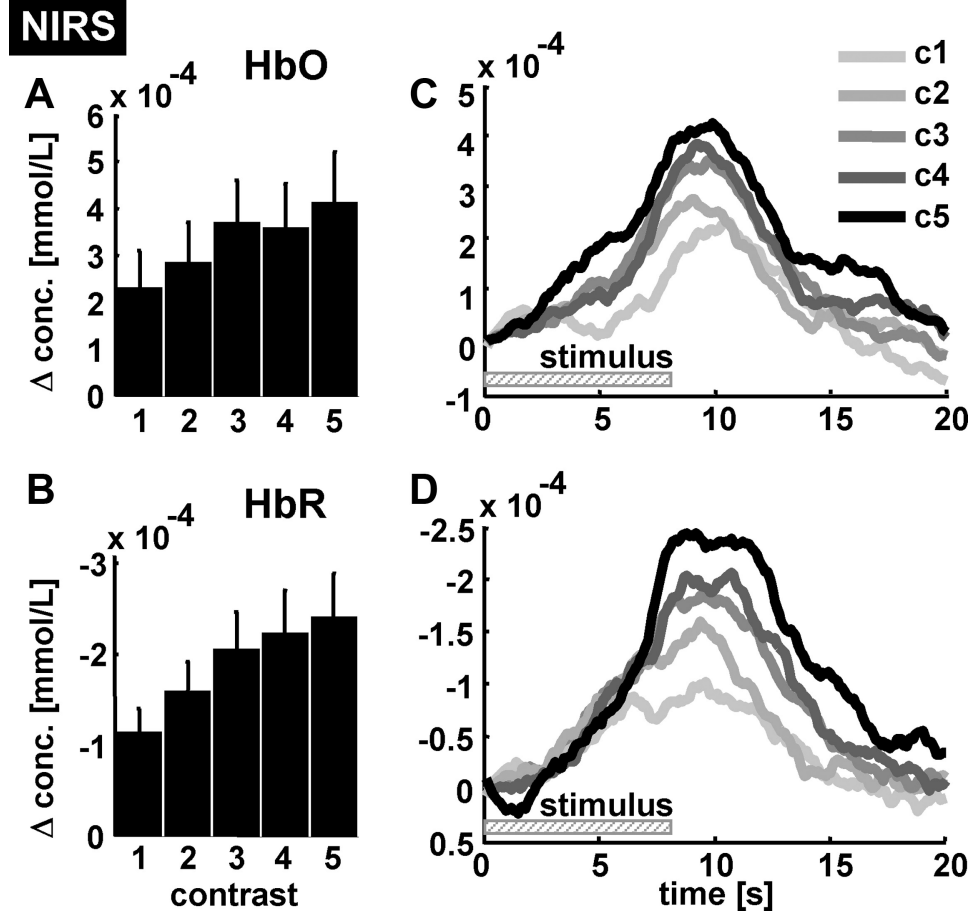

Figure 4. Grand average hemodynamic response to contrast variation. Relative concentration changes of $\mathrm{HbO}$ and $\mathrm{HbR}$ to contrast variation were derived from occipital ROI (pooled probes colocated to the electrodes 01 and 02 ). $A, B$, Mean changes in between contrast level and hemodynamic response magnitude. Note that $\mathrm{HbR}$ changes are plotted in an inverted manner (negain $\mathrm{HbR}$ indicates a more rapid washout of $\mathrm{HbR}$ from the activated area (i.e., a larger hemodynamic response). The vertical bars
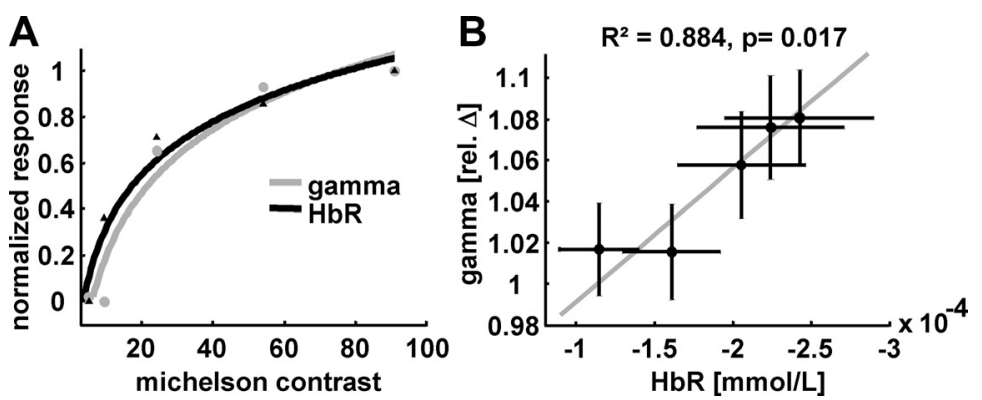

Figure 5. Relationship between contrast, occipital gamma power, and colocated HbR. $A$, A logarithmic model is most suitable to explain the relationship between Michelson contrast and gamma power (gray line) as well as HbR (black line). $\boldsymbol{B}$, When gamma power is plotted against $\mathrm{HbR}$, both parameters reveal a strong linear relationship. The horizontal and vertical bars denote SEM across subjects. The gray line depicts the fitted linear model.

$0.204, p=0.842$; beta: $t_{(11)}=1.736, p=0.11$ ) (supplemental Fig. 4, available at www.jneurosci.org as supplemental material).

The differences in gamma power were closely linked to the hemodynamic response (pooled from occipital probes colocated to $\mathrm{O} 1$ and $\mathrm{O} 2$ electrodes) (Fig. 6C). Larger oxygenation changes were found for fast RT compared with slow RT (Fig. 6D,E) $\left(\mathrm{HbO}, t_{(11)}=2.712, p=0.02 ; \mathrm{HbR}, t_{(11)}=-2.935, p=0.01\right)$. The topography of gamma-band changes shows that the electrophysiological response extends parietally beyond the area sampled by the optical imaging array (Fig. $6 F-H$ ). In analogy to the VEP elicited by the contrast change (supplemental Fig. 3, available at www.jneurosci.org as supplemental material), we averaged the evoked response to velocity changes. The most 

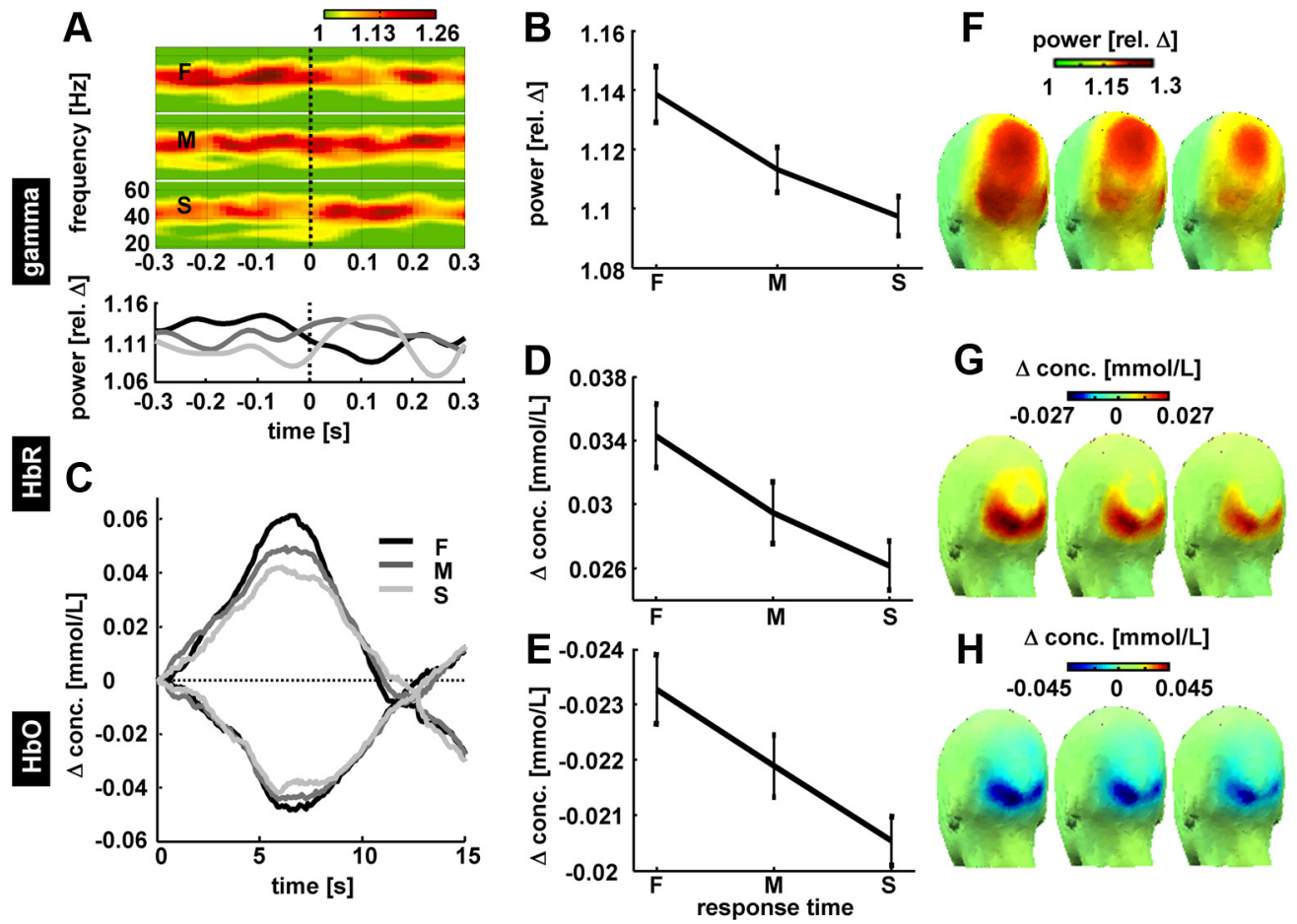

Figure 6. Response variation attributable to changes in internal state-dependent variables (preparedness). Within the strongest contrast ( $(5)$ ), epochs based on velocity change onset were ranked according to the RT, divided in equally large groups of fast (F), medium (M), and slow (S) RTs and averaged across subjects. $A$, Top, Time-frequency representations of gamma power (pooled occipital electrodes 01 and 02 ) for fast, medium, and slow trials depicted for a time window of -0.3 to $0.3 \mathrm{~s}$ after velocity. Note that the gamma power shortly before the change best predicts RT. $A$, Bottom, Time course of gamma activity (same as above but averaged across frequencies $35-70 \mathrm{~Hz}$ ). B, Gamma activity averaged from 200 to $100 \mathrm{~ms}$ before the velocity change for fast, medium, and slow trials shows a clear decrease in gamma activity with increasing RT. Error bars denote SEM. C, Grand average hemodynamic response for fast, medium, and slow trials from occipital ROI (pooled probes correspond to the location of electrodes 01 and 02$)$. D, E, Changes in $\mathrm{HbO}(\boldsymbol{D})$ and changes in $\mathrm{HbR}(\boldsymbol{E})$ for fast, medium, and slow trials. Note that $\mathrm{HbR}$ scaling is inverted (decrease in concentration upward as in Fig. 4). $\boldsymbol{F}-\boldsymbol{H}$, Scalp distribution of gamma activity, $\mathrm{HbO}$, and $\mathrm{HbR}$ for fast (F), medium (M), and slow (S) trials.

pronounced component of the evoked potential peaking $\sim 250$ ms after the velocity change did not systematically vary with reaction time (data not shown).

In sum, the results supply evidence in the human that spontaneous fluctuations in gamma power before the behaviorally relevant stimulus change partly predict reaction time. Moreover, the spontaneous variations in gamma power correlate with the vascular response to the stimulus. It seems noteworthy that the finding with respect to gamma activity closely matches the results from the invasive study on speed of change detection in monkeys [compare Fig. $6 A$ with Womelsdorf et al. (2006), their Fig. 2].

\section{Discussion}

Synchronization of neuronal signaling in the gamma frequency range is a putative neurophysiological mechanism to process stimuli and efficiently integrate a multitude of features (Engel et al., 2001). Therefore, gamma synchronization is suggested to play a key role in the human brain's exceptional capacity to generate and integrate percepts in rapid succession, which is mandatory for most higher-order cognitive function. In the human, we here supply evidence to support two important hypotheses highlighting the central role of gamma-band oscillations for the imaging of cognitive function noninvasively. (1) Gamma band response is augmented with parametric increases in stimulus contrast in the human visual cortex (Siegel et al., 2007). Apart from this "externally" driven modulation, we show that gamma activity before a task-relevant cue (velocity change) determines reaction time, which illustrates internal state dependence of GBR. Thus, GBR can be considered as a hinge between external and internal determinants of the variability in response velocity. (2) Evidence is supplied that GBR is closely related to the vascular response in the human brain. Both GBR attributable to changes in stimulus salience and state-dependent GBR predicting reaction time within a given stimulus contrast are reflected by concomitant hemodynamic changes. Linking GBR to behavior and the vascular response is highly relevant to the neuroimaging of human cognition.

\section{Stimulus-induced GBR modulation}

With respect to the externally modulated GBR, we find a logarithmic relationship between Michelson contrast and gamma activity. Notably, GBR was sustained over the full duration (7 s) of the trial (Fig. $3 A$ ), extending the results of a recent magnetoencephalography (MEG) study (Hoogenboom et al., 2006) using a single contrast over $3 \mathrm{~s}$. The current findings cannot be explained merely by top-down modulation. This is because lower contrasts cause relatively small GBR but necessitate more attentional resources, as indexed by slower reaction time; whereas stronger contrasts cause larger GBR and faster responses. Hence the positive relationship between contrast and GBR indicates a stimulusdriven signal. Comparing human MEG data to monkey data (Logothetis et al., 2001), a previous study reports a linear, as opposed to the logarithmic increase in GBR with contrast that we found (Hall et al., 2005). The difference between the human data reported by Hall et al. and our data may relate to the difference in stimulus presentation (stationary vs moving) and the choice of contrast levels. For the evoked potential on the changes in contrast, which selectively samples the transient response, we also find a logarithmic increase with contrast in the present study (supplemental Fig. 3, available at www.jneurosci.org as supple- 
mental material). In line with our finding, a recent study on transient gamma response shows a nonlinear but steady increase across contrasts in a forced-choice discrimination task (Schadow et al., 2007), whereas in cats, a linear dependence was observed at low and medium contrasts, which became logarithmic with stronger contrast (Tolhurst et al., 1981).

\section{Relationship between contrast and other frequency bands}

Beyond GBR, theta activity also increased during stimulation, whereas alpha and beta power were attenuated. The response in lower bands did not reveal a systematic variation with contrast. This finding is consistent with a number of invasive studies supporting the view that gamma activity rather than lower frequency changes reflect stimulus configurations and task sets (Frien and Eckhorn, 2000; Frien et al., 2000), in particular for visual contrast modulation as shown in the monkey (Henrie and Shapley, 2005). In humans, evidence for sustained GBR in response to parametrically varied stimulus strengths is scarce. Our data suggest that sustained GBR reflects processing and encoding of task relevant information, whereas lower frequency bands are probably less stimulus related.

\section{How is the GBR reflected in the vascular response?}

Theoretically, the augmentation of a specific frequency band may result from either the recruitment of a larger network or phaselocking of preexisting oscillators. Oscillatory activity at higher frequencies involves enhanced activity of GABAergic interneurons (Traub et al., 1996; Hasenstaub et al., 2005) and is thus likely to cause an enhanced metabolic demand (Niessing et al., 2005). In the cat's visual cortex, synchronization rather than neuronal recruitment is altered on midbrain stimulation (Munk et al., 1996). Also, strabismic cats show no difference in cellular discharge on binocular rivalry stimulation, whereas synchrony in the gamma range was exclusive for the perceived, as opposed to the suppressed, monocular stimulus (Fries et al., 1997, 2002). Our results are compatible with the results in cats (Niessing et al., 2005) that a GBR increase is reflected by a hemodynamic increase, following the assumption of enhanced inhibitory activity without reduction of a global excitatory drive. In line with the prediction in animals using BOLD contrast (Logothetis et al., 2001) or optical imaging (Niessing et al., 2005), we find support in humans for a tight relationship between GBR and its hemodynamic counterpart. Recent approaches in humans using complex stimuli relied on a comparison between invasive recordings in neurosurgical patients and the BOLD response in healthy volunteers (Mukamel et al., 2005; Nir et al., 2007). Although limited by interindividual comparison, these approaches also demonstrate a close coupling between both parameters.

\section{State-dependent GBR changes}

GBR also varied within the same contrast, which presumably reflects endogenous fluctuations (e.g., attentional modulation or "the preparedness to respond") (Engel et al., 2001; Schoffelen et al., 2005). We find that fast responses to the velocity change are heralded by enhanced gamma activity before the cue. In the human, this result confirms LFP recordings from area V4 in monkeys showing that GBR predicts reaction time. In a focused attention task, reaction time covaried with GBR several hundred milliseconds before the task-relevant cue (Womelsdorf et al., 2006). Our results show a remarkably similar dynamic and spectral range of the GBR [compare Fig. 6A with Womelsdorf et al. (2006), their Figs. 2, 4C]. Moreover, GBR fluctuations before cue onset elicit a graduated vascular response supplying direct evi- dence that internal state-dependent GBR is detectable by vascular-based imaging techniques. This is noteworthy since synchronization driven by some distant network might not enhance the local metabolic demand. With regard to the optical imaging study in cats, we suggest that tightly coupled stimulus-independent GBR and hemodynamic response variability (Niessing et al., 2005) would correlate to internal state variables when tested. In animals and humans, the predictive value of gamma augmentation for behavior has been addressed in visuomotor and other tasks (Gonzalez Andino et al., 2005; Womelsdorf et al., 2006). An advantage of our approach is that stimulus salience (contrast) and task relevant cue (velocity) are independently modulated, allowing testing of both determinants for GBR variations within the same experiment. A recent study in monkeys investigated the relationship between LFP and multiunit activity in visual MT area and demonstrated that LFP is linked to "choice probability" in a speed detection task. This correlation only holds for higher frequencies $(>40 \mathrm{~Hz})$, whereas for lower frequencies an anticorrelation is found (Liu and Newsome, 2006). Although on a much coarser scale, we observe a similar predictive value of GBR in the human visual cortex and supply evidence for a close relationship between local neuronal activity and the vascular response. In an elegant design, it has been recently shown that nonattentional internal modulation, namely decision bias and awareness, are reflected by prestimulus gamma synchronization (Wyart and Tallon-Baudry, 2009). The temporal window of gamma-band activity (300-100 ms before the stimulus), which is shown to correlate with decision bias, is similar to the time window (200-100 ms before the velocity change) in which we find the state-dependent predictive gamma modulation for the behavioral response. Concerning the hemodynamic based imaging, another recent study (Hesselmann et al., 2008) demonstrated that prestimulus BOLD changes in the fusiform face area correlate with the bias to perceive faces as opposed to a vase in a classical Rubin figure. The predictive value of prestimulus gamma synchronization and oxygenation changes in the present study may therefore encompass even more specific aspects of the behavioral response. Additional studies in combined neurovascular approaches are needed to clarify whether the here-proposed tight coupling between GBR and vascular response also holds true for differential ingredients of internal modulations.

\section{Cerebral origin of gamma-band response}

Our study supplies evidence for the coupling between GBR and vascular response. The recent heated discussion (Fries et al., 2008; Yuval-Greenberg et al., 2008; Melloni et al., 2009) as to the cerebral origin of GBR in noninvasive recordings is not a focus of the paper. Nonetheless, the cerebral origin of GBR changes is a prerequisite for the inference drawn from the results. The registered ocular electrodes revealed no systematic changes with contrast variation (supplemental Fig. 2, available at www.jneurosci.org as supplemental material). Although we did not record miniature saccades by an eye-tracking system, we regard the measured gamma activity as a cerebral signal and do not consider them liable to stem from event-related microsaccades as has been shown for transient bursts of "induced" gamma (YuvalGreenberg et al., 2008) since (1) GBR is sustained for the entire stimulation period ( $7 \mathrm{~s}$ ) as opposed to the transient GBR response reported by a number of studies. Interestingly, in a pilot study, we found that GBR increase is stable over stimulation periods of up to $70 \mathrm{~s}$ (data not shown). (2) GBR increases parametrically with contrast strength at parieto-occipital electrodes, and (3) GBR is closely coupled to the regional hemodynamic response, which is 
completely unsusceptible to ocular movement. Beyond that, our state-dependent GBR results are strikingly similar to results in monkeys (Womelsdorf et al., 2006) and confirm comparable contrast-dependent LFP modulations in the gamma range in other invasive studies (Henrie and Shapley, 2005).

\section{Conclusion}

Oscillations in the gamma range must be regarded as a multifunctional encoding mechanism forming the basis for visual feature integration (Gray and McCormick, 1996) and object recognition (Tallon-Baudry and Bertrand, 1999; Mima et al., 2001). Therein, higher cognitive factors such as attention (Gruber et al., 1999; Fries et al., 2001; Taylor et al., 2005) and anticipation (Lee, 2003; Fitzgibbon et al., 2004) have modulatory influences on the same encoding mechanism. Our data show that both exogenously and endogenously triggered fluctuations in the gamma range are mirrored in a vascular response and are hence accessible to vascularbased functional imaging.

\section{References}

Arieli A, Sterkin A, Grinvald A, Aertsen A (1996) Dynamics of ongoing activity: explanation of the large variability in evoked cortical responses. Science 273:1868-1871.

Boynton GM, Engel SA, Glover GH, Heeger DJ (1996) Linear systems analysis of functional magnetic resonance imaging in human V1. J Neurosci 16:4207-4221.

Caesar K, Gold L, Lauritzen M (2003) Context sensitivity of activitydependent increases in cerebral blood flow. Proc Natl Acad Sci U S A 100:4239-4244.

Carrasco M, Ling S, Read S (2004) Attention alters appearance. Nat Neurosci 7:308-313.

Eckhorn R, Bauer R, Jordan W, Brosch M, Kruse W, Munk M, Reitboeck HJ (1988) Coherent oscillations: a mechanism of feature linking in the visual cortex? Multiple electrode and correlation analyses in the cat. Biol Cybern 60:121-130.

Engel AK, Fries P, Singer W (2001) Dynamic predictions: oscillations and synchrony in top-down processing. Nat Rev Neurosci 2:704-716.

Fitzgibbon SP, Pope KJ, Mackenzie L, Clark CR, Willoughby JO (2004) Cognitive tasks augment gamma EEG power. Clin Neurophysiol 115:1802-1809.

Frien A, Eckhorn R (2000) Functional coupling shows stronger stimulus dependency for fast oscillations than for low-frequency components in striate cortex of awake monkey. Eur J Neurosci 12:1466-1478.

Frien A, Eckhorn R, Bauer R, Woelbern T, Gabriel A (2000) Fast oscillations display sharper orientation tuning than slower components of the same recordings in striate cortex of the awake monkey. Eur J Neurosci 12:1453-1465.

Fries P, Roelfsema PR, Engel AK, König P, Singer W (1997) Synchronization of oscillatory responses in visual cortex correlates with perception in interocular rivalry. Proc Natl Acad Sci U S A 94:12699-12704.

Fries P, Reynolds JH, Rorie AE, Desimone R (2001) Modulation of oscillatory neuronal synchronization by selective visual attention. Science 291:1560-1563.

Fries P, Schröder JH, Roelfsema PR, Singer W, Engel AK (2002) Oscillatory neuronal synchronization in primary visual cortex as a correlate of stimulus selection. J Neurosci 22:3739-3754.

Fries P, Scheeringa R, Oostenveld R (2008) Finding gamma. Neuron 58:303-305.

Goldman RI, Stern JM, Engel J Jr, Cohen MS (2002) Simultaneous EEG and fMRI of the alpha rhythm. Neuroreport 13:2487-2492.

Gonzalez Andino SL, Michel CM, Thut G, Landis T, Grave de Peralta R (2005) Prediction of response speed by anticipatory high-frequency (gamma band) oscillations in the human brain. Hum Brain Mapp 24:50-58.

Gray CM, McCormick DA (1996) Chattering cells: superficial pyramidal neurons contributing to the generation of synchronous oscillations in the visual cortex. Science 274:109-113.

Gray CM, Singer W (1989) Stimulus-specific neuronal oscillations in orientation columns of cat visual cortex. Proc Natl Acad Sci USA $86: 1698-1702$.
Gray CM, König P, Engel AK, Singer W (1989) Oscillatory responses in cat visual cortex exhibit inter-columnar synchronization which reflects global stimulus properties. Nature 338:334-337.

Gruber T, Müller MM, Keil A, Elbert T (1999) Selective visual-spatial attention alters induced gamma band responses in the human EEG. Clin Neurophysiol 110:2074-2085.

Hall SD, Holliday IE, Hillebrand A, Singh KD, Furlong PL, Hadjipapas A, Barnes GR (2005) The missing link: analogous human and primate cortical gamma oscillations. Neuroimage 26:13-17.

Hasenstaub A, Shu Y, Haider B, Kraushaar U, Duque A, McCormick DA (2005) Inhibitory postsynaptic potentials carry synchronized frequency information in active cortical networks. Neuron 47:423-435.

Henrie JA, Shapley R (2005) LFP power spectra in V1 cortex: the graded effect of stimulus contrast. J Neurophysiol 94:479-490.

Hesselmann G, Kell CA, Eger E, Kleinschmidt A (2008) Spontaneous local variations in ongoing neural activity bias perceptual decisions. Proc Natl Acad Sci U S A 105:10984-10989.

Hoogenboom N, Schoffelen JM, Oostenveld R, Parkes LM, Fries P (2006) Localizing human visual gamma-band activity in frequency, time and space. Neuroimage 29:764-773.

Huppert TJ, Hoge RD, Diamond SG, Franceschini MA, Boas DA (2006) A temporal comparison of BOLD, ASL, and NIRS hemodynamic responses to motor stimuli in adult humans. Neuroimage 29:368-382.

Kleinschmidt A, Obrig H, Requardt M, Merboldt KD, Dirnagl U, Villringer A, Frahm J (1996) Simultaneous recording of cerebral blood oxygenation changes during human brain activation by magnetic resonance imaging and near-infrared spectroscopy. J Cereb Blood Flow Metab 16:817-826.

Koch SP, Steinbrink J, Villringer A, Obrig H (2006) Synchronization between background activity and visually evoked potential is not mirrored by focal hyperoxygenation: implications for the interpretation of vascular brain imaging. J Neurosci 26:4940-4948.

Lee D (2003) Coherent oscillations in neuronal activity of the supplementary motor area during a visuomotor task. J Neurosci 23:6798-6809.

Liu J, Newsome WT (2006) Local field potential in cortical area MT: stimulus tuning and behavioral correlations. J Neurosci 26:7779-7790.

Logothetis NK, Pauls J, Augath M, Trinath T, Oeltermann A (2001) Neurophysiological investigation of the basis of the fMRI signal. Nature 412:150-157.

Martínez-Trujillo J, Treue S (2002) Attentional modulation strength in cortical area MT depends on stimulus contrast. Neuron 35:365-370.

Melloni L, Schwiedrzik CM, Wibral M, Rodriguez E, Singer W (2009) Response to: Yuval-Greenberg et al., "Transient Induced Gamma-Band Response in EEG as a Manifestation of Miniature Saccades." Neuron 58, 429-441. Neuron 62:8-10; author reply 10-12.

Mima T, Oluwatimilehin T, Hiraoka T, Hallett M (2001) Transient interhemispheric neuronal synchrony correlates with object recognition. J Neurosci 21:3942-3948.

Moosmann M, Ritter P, Krastel I, Brink A, Thees S, Blankenburg F, Taskin B, Obrig H, Villringer A (2003) Correlates of alpha rhythm in functional magnetic resonance imaging and near infrared spectroscopy. Neuroimage 20:145-158.

Mukamel R, Gelbard H, Arieli A, Hasson U, Fried I, Malach R (2005) Coupling between neuronal firing, field potentials, and FMRI in human auditory cortex. Science 309:951-954.

Munk MH, Roelfsema PR, König P, Engel AK, Singer W (1996) Role of reticular activation in the modulation of intracortical synchronization. Science 272:271-274.

Niessing J, Ebisch B, Schmidt KE, Niessing M, Singer W, Galuske RA (2005) Hemodynamic signals correlate tightly with synchronized gamma oscillations. Science 309:948-951.

Nir Y, Fisch L, Mukamel R, Gelbard-Sagiv H, Arieli A, Fried I, Malach R (2007) Coupling between neuronal firing rate, gamma LFP, and BOLD fMRI is related to interneuronal correlations. Curr Biol 17:1275-1285.

Obrig H, Villringer A (2003) Beyond the visible-imaging the human brain with light. J Cereb Blood Flow Metab 23:1-18.

Reynolds JH, Chelazzi L (2004) Attentional modulation of visual processing. Annu Rev Neurosci 27:611-647.

Reynolds JH, Desimone R (2003) Interacting roles of attention and visual salience in V4. Neuron 37:853-863.

Schadow J, Lenz D, Thaerig S, Busch NA, Fründ I, Rieger JW, Herrmann CS 
(2007) Stimulus intensity affects early sensory processing: visual contrast modulates evoked gamma-band activity in human EEG. Int J Psychophysiol 66:28-36.

Schoffelen JM, Oostenveld R, Fries P (2005) Neuronal coherence as a mechanism of effective corticospinal interaction. Science 308:111-113.

Siegel M, Donner TH, Oostenveld R, Fries P, Engel AK (2007) Highfrequency activity in human visual cortex is modulated by visual motion strength. Cereb Cortex 17:732-741.

Sirotin YB, Das A (2009) Anticipatory haemodynamic signals in sensory cortex not predicted by local neuronal activity. Nature 457:475-479.

Tallon-Baudry C, Bertrand O (1999) Oscillatory gamma activity in humans and its role in object representation. Trends Cogn Sci 3:151-162.

Taylor K, Mandon S, Freiwald WA, Kreiter AK (2005) Coherent oscillatory activity in monkey area $\mathrm{v} 4$ predicts successful allocation of attention. Cereb Cortex 15:1424-1437.
Tolhurst DJ, Movshon JA, Thompson ID (1981) The dependence of response amplitude and variance of cat visual cortical neurones on stimulus contrast. Exp Brain Res 41:414-419.

Traub RD, Whittington MA, Stanford IM, Jefferys JG (1996) A mechanism for generation of long-range synchronous fast oscillations in the cortex. Nature 383:621-624.

Womelsdorf T, Fries P, Mitra PP, Desimone R (2006) Gamma-band synchronization in visual cortex predicts speed of change detection. Nature 439:733-736.

Wyart V, Tallon-Baudry C (2009) How ongoing fluctuations in human visual cortex predict perceptual awareness: baseline shift versus decision bias. J Neurosci 29:8715-8725.

Yuval-Greenberg S, Tomer O, Keren AS, Nelken I, Deouell LY (2008) Transient induced gamma-band response in EEG as a manifestation of miniature saccades. Neuron 58:429-441. 